TRANSACTIONS OF THE

AMERICAN MATHEMATICAL SOCIETY

Volume 357, Number 12, Pages 5031-5050

S 0002-9947(05)03656-1

Article electronically published on March 18, 2005

\title{
AN ALGEBRAIC APPROACH TO MULTIRESOLUTION ANALYSIS
}

\author{
RICHARD FOOTE
}

\begin{abstract}
The notion of a weak multiresolution analysis is defined over an arbitrary field in terms of cyclic modules for a certain affine group ring. In this setting the basic properties of weak multiresolution analyses are established, including characterizations of their submodules and quotient modules, the existence and uniqueness of reduced scaling equations, and the existence of wavelet bases. These results yield some standard facts on classical multiresolution analyses over the reals as special cases, but provide a different perspective by not relying on orthogonality or topology. Connections with other areas of algebra and possible further directions are mentioned.
\end{abstract}

\section{INTRODUCTION}

The theory of multiresolution analyses and wavelets, which has its roots in papers by Mallat, Grossmann, Morlet, Paul, Daubechies and others in the 1980's, has flourished in recent years, and its uses have been numerous and profound (see 6, [11, [13, [16]). In particular, the theory has become a cornerstone of modern signal processing, with applications permeating areas such as digital image processing, data compression, pattern recognition, data mining, and the like. The multiresolution analysis framework, which is normally viewed in the larger context of Fourier analysis, provides, in particular, methods for extracting local information from data defined over a global spatial or temporal domain. Classical Fourier analysis via exponential Fourier series and transforms on the real line and on intervals is rooted in the representation theory of the unit circle group and its subgroups. Indeed generalizations, new applications, and deeper understandings of Fourier analysis were often achieved from the group-theoretic perspective. For instance, the Discrete Fourier Transform and Fast Fourier Transform are now clearly understood within the context of representations of finite cyclic groups. Generalizations and concomitant applications of these transforms based on other (possibly non-abelian) finite or compact groups or over other fields are emerging in greater number in the literature (see, in particular, 11, [2, 5], 15], 17], 18, [19, 20], 23] and the references therein; see also the introduction to [9] for a survey).

Likewise, it is known that a multiresolution analysis in $L^{2}(\mathbb{R})$ results from an action of a subgroup of the affine group on this space. The purpose of this paper is to pursue this representation-theoretic perspective by axiomatizing its essential

Received by the editors March 7, 2003 and, in revised form, January 14, 2004 and March 5, 2004 .

2000 Mathematics Subject Classification. Primary 20C99; Secondary 42C40.

Key words and phrases. Multiresolution analysis, wavelets.

This work was partially supported by an AFOSR/NM grant. 
algebraic features. This approach, which in some respects is simpler and more general than the original theory, applies over an arbitrary field and does not require an orthogonal form or a topology on the underlying representation space (so, in particular, it may be utilized in $L^{p}$-spaces other than $L^{2}$ ). From this vantage we give new proofs of some of the basic classical properties of multiresolution analyses and wavelets. For example, we see precisely how certain scaling functions lead to a wavelet basis over any field. We also mention possible connections to other areas of algebra and number theory, and suggest some avenues for further investigation to stimulate both theoretical and applications oriented research.

More specifically, the paper is organized as follows. In Section 2 we review the definition of a multiresolution analysis and extract some of its features to define a weak multiresolution analysis (WMA) over any field. In Section 3 we cast some aspects of WMAs into a ring-theoretic framework; this initiates some algebraicgeometric methods that are used subsequently, and also suggests connections between multiresolution analyses and other areas. Section 4 begins the main results of the paper: a classification of weak multiresolution analyses over arbitrary fields, and a determination of their submodules and quotient modules. In Section 5 we prove that every infinite-dimensional WMA has a "wavelet basis," and possesses a unique "reduced scaling equation" that characterizes it up to module isomorphism. In Section 6 we suggest some lines for possible further investigation.

This paper is intentionally self-contained and elementary, making it accessible to a general audience. Other papers that mention or pursue some representationtheoretic aspects of multiresolution analysis include [3], 6], [8, [9] [11, 12], 13], 21], 22], 26], 27], but these complement rather than overlap substantially with this paper. The work of Chirikjian and his collaborators, 5], [15], uses the tools of harmonic analysis on the affine group and motion groups in various pattern recognition problems.

\section{WEAK MULTIRESOLUTION ANALYSES: DEFINITIONS AND PRELIMINARY RESULTS}

Following standard references such as [6], 14], 25], a multiresolution analysis is a vector subspace $V$ of $L^{2}(\mathbb{R})$ together with subspaces $V_{i}$ of $V$ for each $i \in \mathbb{Z}$ satisfying the following axioms:

MR1. For each $f \in V, f(x) \in V_{i}$ if and only if $f(2 x) \in V_{i-1}$.

MR2. The subspaces $V_{i}$ are nested: $\cdots V_{2} \subseteq V_{1} \subseteq V_{0} \subseteq V_{-1} \subseteq V_{-2} \cdots$.

MR3. $\bigcup_{i \in \mathbb{Z}} V_{i}=V$ and $V$ is dense in $L^{2}(\mathbb{R})$.

MR4. $\bigcap_{i \in \mathbb{Z}} V_{i}=0$.

MR5. If $f(x) \in V_{0}$, then $f(x-k) \in V_{0}$ for all $k \in \mathbb{Z}$.

MR6. There is a function $\phi(x)$ such that $\{\phi(x-k) \mid k \in \mathbb{Z}\}$ is an orthonormal basis of $V_{0}$.

Axioms 1 and 2 specify a "multiresolution" decomposition of $V$ "scaled" by factors of two, where $f(2 x)$ is thought of as a "finer" version of $f(x)$. Axioms 3 and 4 are standard in approximation theory. From the so-called scaling function $\phi$, and assuming the subspaces $V_{i}$ are closed in $L^{2}(\mathbb{R})$, these axioms easily lead to a mother wavelet, $\psi$, whose scaled and translated versions, $\left\{\psi\left(2^{i} x-j\right) \mid i, j \in \mathbb{Z}\right\}$, span a dense subset of $L^{2}(\mathbb{R})$, hence give "wavelet series" for square-integrable functions (see Section 5). The term "basis" in axiom 6 refers to a Hilbert space basis; however 
we shall restrict our attention to the class of multiresolution analyses where every element of $V_{0}$ is a (finite) linear combination of the translates of $\phi$. This class includes all multiresolution analyses where the scaling function and corresponding wavelets have compact support.

We extract some essential features of such a multiresolution analysis by first observing that if $Y$ is any nonempty set, there is a left group action by the real affine group on the set of all functions $f: \mathbb{R} \rightarrow Y$ by

$$
\left(\begin{array}{ll}
a & b \\
0 & 1
\end{array}\right) f(x)=f\left(\frac{x-b}{a}\right) \quad \text { for }\left(\begin{array}{ll}
a & b \\
0 & 1
\end{array}\right) \in G L_{2}(\mathbb{R}) .
$$

In the case of real-valued functions, this action is $\mathbb{R}$-linear and restricts to an action on various subspaces such as $L^{2}(\mathbb{R})$ or the space of continuous functions. We single out some relevant subgroups and elements of this affine group:

$$
\begin{gathered}
G=\left\{\left(\begin{array}{cc}
2^{a} & b \\
0 & 1
\end{array}\right) \mid a \in \mathbb{Z}, b \in \mathbb{Z}[1 / 2]\right\} \\
\tau=\left(\begin{array}{ll}
1 & 1 \\
0 & 1
\end{array}\right), \quad \sigma=\left(\begin{array}{ll}
2 & 0 \\
0 & 1
\end{array}\right), \quad B_{j}=\left\{\left(\begin{array}{cc}
1 & 2^{j} b \\
0 & 1
\end{array}\right) \mid b \in \mathbb{Z}\right\} .
\end{gathered}
$$

Under the action of $G$ on $L^{2}(\mathbb{R})$ we easily see that some of the essential features that define a multiresolution analysis $V$ with scaling function $\phi$ can now be couched in an algebraic framework:

MRa. $V$ is a cyclic $\mathbb{R} G$-module with generator $\phi$.

MRb. The "zero scale" subspace $V_{0}$ is the cyclic $\mathbb{R} B_{0}$-submodule of $V$ generated by $\phi$.

MRc. $V_{i}=\sigma^{i} V_{0}$ and $V_{i} \subseteq \sigma^{-1} V_{i}$ for all $i \in \mathbb{Z}$.

The fundamental perspective of this paper is to transport these properties to an arbitrary field, and to unravel consequences of this abstraction, recovering many of the properties of multiresolution analyses over $\mathbb{R}$ in the process. We note that the above properties distinguish $B_{0}$ from its conjugate subgroups $B_{i}$, so we also wish to work with a formulation which avoids this asymmetry.

Definition. Let $F$ be any field and let $G$ be the group in (2.1) above. For $j \in \mathbb{Z}$, a nonzero $F G$-module $V$ is called a weak multiresolution analysis of level $j$ over $F$ with respect to $v_{0}$ if $V$ is generated as a cyclic $F G$-module by $v_{0}$, and if we let $V_{j, 0}=$ $F B_{j} v_{0}$, then $\sigma^{i} V_{j, 0} \subseteq \sigma^{i-1} V_{j, 0}$ for all $i \in \mathbb{Z}$. We say $V$ is a weak multiresolution analysis (or WMA) over $F$ with respect to $v_{0}$ if it is a weak multiresolution analysis with respect to $v_{0}$ of level $j$ for some $j \in \mathbb{Z}$.

We say $V$ is a WMA if it is a WMA with respect to some generator $v_{0}$, and we call $v_{0}$ a scaling function for $V$. Thus a "classical" multiresolution analysis discussed above is a weak multiresolution analysis over $\mathbb{R}$ of level zero. We shall see examples where $V$ is a WMA with respect to some generator $v_{0}$ but not with respect to some other cyclic generator, so explicit specification of a distinguished generator is often required.

For the remainder of this section $F$ is an arbitrary field, $G$ is the group in (2.1), and $V$ is a cyclic $F G$-module with generator $v_{0}$. Fix some $j \in \mathbb{Z}$ and let $V_{j, 0}=F B_{j} v_{0}$ and $V_{j, i}=\sigma^{i} V_{j, 0}$. We introduce some additional notation used 
throughout the paper:

$$
\tau_{j}=\left(\begin{array}{cc}
1 & 2^{j} \\
0 & 1
\end{array}\right), \quad B=\left\{\left(\begin{array}{ll}
1 & b \\
0 & 1
\end{array}\right) \mid b \in \mathbb{Z}[1 / 2]\right\} .
$$

Observe that the following relations hold:

(i) $\tau_{j}^{2}=\tau_{j+1}$ and $\sigma \tau_{j} \sigma^{-1}=\tau_{j+1}$, so $\sigma \tau_{j}=\tau_{j}^{2} \sigma$.

(ii) $\tau=\tau_{0}$ and $B_{0}=\langle\tau\rangle$.

(iii) $\tau_{j}=\sigma^{j} \tau \sigma^{-j}$ and $B_{j}=\sigma^{j} B_{0} \sigma^{-j} \cong \mathbb{Z}$ for all $j \in \mathbb{Z}$.

(iv) $\sigma^{i} B_{j} \sigma^{-i}=B_{j+i}$.

(v) $\cdots B_{2} \subset B_{1} \subset B_{0} \subset B_{-1} \subset B_{-2} \subset \cdots$ and $\bigcup_{i=0}^{\infty} B_{-i}=B$.

(vi) $B \cong \mathbb{Z}[1 / 2]$ and $B$ is a 2-divisible, locally cyclic group (i.e., every finitely generated subgroup is cyclic).

(vii) $G=\langle\tau, \sigma\rangle, B$ is a characteristic subgroup of $G$, and $G$ is the split extension of $\langle\sigma\rangle$ by $B$; all complements to $B$ in $G$ are conjugate to $\langle\sigma\rangle$ (i.e., $\left.H^{1}(\langle\sigma\rangle, B)=0\right)$.

(viii) $B=G^{\prime}$, and $N$ is a nontrivial normal subgroup of $G$ if and only if $B \subseteq N$.

(ix) Each $V_{j, i}$ is an $F B_{j+i}$-module, and hence is an $F B_{k}$-module for any $k \geq j+i$ as well.

(x) Let $w_{0} \in V$, let $w_{-1}=\sigma^{-1} w_{0}$, and let $g(X)$ be any $F$-linear combination of integer powers of the variable $X$. Then $\sigma^{-1} g\left(\tau_{j}\right) w=g\left(\tau_{j-1}\right) w_{-1}$.

In the special case where $V$ is a classical multiresolution analysis over $\mathbb{R}$, the last observation is a restatement of the fact that $f(2 x-b)=f(2(x-b / 2))$.

Proposition 2.1. In the notation of this section the following are equivalent:

(1) $V$ is a WMA of level $j$ with respect to the generator $v_{0}$.

(2) $V_{j, 0} \subseteq V_{j,-1}$.

(3) $V_{j, i} \subseteq V_{j, i-1}$ for some $i \in \mathbb{Z}$.

(4) For some $\beta \in F B_{j}$ we have $v_{0}=\sigma^{-1} \beta v_{0}$.

(5) There is an isomorphism $\Phi: F G / L \rightarrow V$ of left $F G$-modules with $\Phi(1)=v_{0}$ such that $L$ is a left ideal of $F G$ containing an element $\sigma-g\left(\tau_{j}\right)$, where $g\left(\tau_{j}\right)=\tau_{j}^{-n} f\left(\tau_{j}\right)$ for some polynomial $f$ with coefficients from $F$ and nonnegative integer $n$.

Proof. Since $\sigma$ is a unit in $F G$, repeated applications of integer powers of $\sigma$ to $V_{j, 0}$ gives the equivalence of (1), (2) and (3). Since $V_{j, 0}$ is a cyclic $F B_{j}$-module with generator $v_{0}$ and $V_{j,-1}$ is an $F B_{j}$-module and is the image of $V_{j, 0}$ under $\sigma^{-1}$, we have $V_{j, 0} \subseteq V_{j,-1}$ if and only if $v_{0} \in V_{j,-1}$. This gives the equivalence of (3) and (4). Since $V$ is a cyclic $F G$-module there is an $F G$-module homomorphism $\Phi: F G \rightarrow V$ with $\Phi(1)=v_{0}$ such that $\operatorname{ker} \Phi$ is the left ideal that annihilates the element $v_{0}$. By (4), $V$ is a WMA of level $j$ if and only if $v_{0}=\sigma^{-1} \tau_{j}^{-n} f\left(\tau_{j}\right) v_{0}$, where the group ring element $\beta$ is written as a polynomial in $\tau_{j}$ with the negative powers factored out. Thus $V$ is a WMA of level $j$ if and only if $1-\sigma^{-1} \tau_{j}^{-n} f\left(\tau_{j}\right) \in L$. Multiplying this group ring element on the left by the unit $\sigma$ now gives the equivalence of (5) with (4).

In the classical theory of multiresolution analyses over $\mathbb{R}$ (of level zero) the relation in (4) or (5) is called the scaling or dilation equation, and is usually written 
as

$$
\phi(x)=\sqrt{2} \sum_{k=-N}^{M} h_{k} \phi(2 x-k), \quad h_{k} \in \mathbb{R} .
$$

In a multiresolution of level $j$ we would allow the translates $k$ in (2.2) to lie in the group $2^{j} \mathbb{Z}$. We shall see that if $V$ is an infinite-dimensional WMA over any field, then the corresponding scaling equation uniquely determines $V$ (up to $F G$-module isomorphism).

Corollary 2.2. If $V$ is a WMA of level $j$ with respect to $v_{0}$, then $V$ is a WMA of level $k$ with respect to $v_{0}$ for all $k \leq j$. Furthermore, any cyclic $F G$-module $V$ generated by $v_{0}$ is a WMA with scaling function $v_{0}$ if and only if $v_{0}=\sigma^{-1} \beta v_{0}$ for some $\beta \in F B$.

Proof. These assertions are immediate from Proposition 2.1(4) since $B_{j} \subseteq B_{k}$ for all $k \leq j$, and $B$ is the union of the $B_{j}$.

Corollary 2.3. Let $V$ be a WMA. Then $V$ is either a cyclic FB-module or it is not finitely generated over $F B$.

Remark. The examples below show that both possibilities occur.

Proof. Assume $V$ is finitely generated over $F B$ by $v_{1}, \ldots, v_{n}$. Pick $N \in \mathbb{Z}$ so that $v_{i} \in V_{N}$ for all $i$, and let $v_{N}=\sigma^{N} v_{0}$. Then $v_{1}, \ldots, v_{n} \in F B_{N} v_{N} \subseteq F B v_{N}$, so $v_{N}$ generates $V$ as a cyclic $F B$-module.

Corollary 2.4. Let $V$ be a WMA of level $j$ with respect to $v_{0}$. For any FG-module $W$ and $F G$-module homomorphism $\Phi: V \rightarrow W$, the image, $\Phi(V)$, is a WMA of level $j$ with respect to $\Phi\left(v_{0}\right)$.

Proof. This is immediate from the equivalence of (1) and (4) in Proposition 2.1.

Remarks. 1. Translation: If $v_{0}$ is a scaling function at level $j$, write its scaling equation as $v_{0}=\sigma^{-1} \tau_{j}^{-n} f\left(\tau_{j}\right) v_{0}$ for some integer $n$ and some $f(X) \in F[X]$ with $f(0) \neq 0$. Replacing $v_{0}$ by $u_{0}=\tau_{j}^{n} v_{0}$ results in a scaling equation $u_{0}=\sigma^{-1} f\left(\tau_{j}\right) u_{0}$. Clearly $u_{0}$ is an $F G$-module generator for $V$ as well. Thus, in particular, we lose no generality in assuming that the scaling equation for some scaling function $v_{0}$ has no "denominators" (negative powers of $\tau_{j}$ ). For $v_{0}$ and $f$ as above we call $f$ the scaling polynomial and call the degree of this "normalized" polynomial $f$ the degree of the scaling function $v_{0}$.

2. Rescaling: Let $V$ be a WMA of level $j$ with scaling function $v_{j, 0}$ and scaling equation $v_{j, 0}=\sigma^{-1} f\left(\tau_{j}\right) v_{j, 0}$ for some $f(X) \in F[X]$. Then $V$ is also a WMA of level zero with scaling function $v_{0}=\sigma^{-j} v_{j, 0}=v_{j,-j}$ and scaling equation (of level zero) $v_{0}=\sigma^{-1} f(\tau) v_{0}$. Thus by replacing the scaling function we may rescale from level $j$ to level zero (or vice versa) without changing the scaling polynomial $f(X)$.

Note that naïvely viewing a given scaling function at a higher level does change its scaling equation (as well as the scaling subspaces). For example, $v_{0}$ at level zero with scaling equation $v_{0}=\sigma^{-1} f(\tau) v_{0}$ has scaling equation $v_{0}=\sigma^{-1} f\left(\tau_{-1}^{2}\right) v_{0}$ if we let $v_{0}$ generate $V$ as a level -1 WMA (i.e., the scaling polynomial is now $f\left(X^{2}\right)$ ). However, we may "rescale" from level $j$ to level zero while keeping the same scaling 
function and scaling polynomial as follows. Define a new $F G$-module structure on $V$ by calling $V^{(-j)}=V$, and making $V^{(-j)}$ into an $F G$-module by

$$
\gamma \cdot v=\sigma^{j} \gamma \sigma^{-j}(v), \quad \text { for all } \gamma \in F G .
$$

This makes $V^{(-j)}$ into a WMA of level zero with scaling function $v_{j, 0}$ and scaling equation $\sigma \cdot v_{j, 0}=f(\tau) \cdot v_{j, 0}$. Moreover, the map $V \rightarrow V^{(-j)}$ given by $v \mapsto \sigma^{j} v=$ $\sigma^{j} \cdot v$ is an isomorphism of $F G$-modules. These observations show that we lose no generality in assuming all WMAs are of level zero.

Examples. 1. Principal WMAs over $F$ : Let $L$ be the principal left ideal of $F G$ generated by $\sigma-c$ for some nonzero $c \in F$. We call $V(c)=F G / L$ a principal $W M A$ over $F$ with scaling function $v_{0}=1+L$. Let $V=V(c)$, so that $V$ is the regular representation for $F B$ (a free rank $1 F B$-module) and is induced from the 1-dimensional representation of $\langle\sigma\rangle$ with eigenvalue $c$. Explicitly, there is a basis $\left\{v_{i}\right\}$ of $V$ indexed by the elements of $\mathbb{Z}[1 / 2]$ such that $V$ is a WMA of level zero with respect to $v_{0}$, where $\tau\left(v_{j}\right)=v_{j+1}$ and $\sigma\left(v_{j}\right)=c v_{2 j}$, for all $j \in \mathbb{Z}[1 / 2]$. With respect to this basis, $V_{0, i}$ is the span of the vectors $v_{2^{i} n}$ for $n \in \mathbb{Z}$, and the scaling equation is $v_{0}=\sigma^{-1} c v_{0}$.

Note that $u_{0}=v_{\frac{1}{2}}$ is clearly another generator of $V$ as a cyclic $F G$-module. Let $U_{0}=F B_{0} u_{0}=\operatorname{Span}\left\{v_{n+\frac{1}{2}} \mid n \in \mathbb{Z}\right\}$. Thus $\sigma u_{0}=v_{1} \notin U_{0}$, so $V$ is not a WMA of level zero with respect to $u_{0}$, although $V$ is a WMA of level -1 with respect to $u_{0}$.

Note that when $F$ has characteristic $\neq 2$, then $w=v_{0}+v_{1}$ is a generator for $V$ as an $F G$-module, but is not a scaling function at any level: $\sigma w=v_{0}+v_{2} \notin F B w$. In particular, $F G w \neq F B w$.

If $c_{1} \neq c_{2}$, then $V\left(c_{1}\right)$ and $V\left(c_{2}\right)$ are nonisomorphic $F G$-modules since each has a unique 1-dimensional eigenspace for $\sigma$ with eigenvalues $c_{1}$ and $c_{2}$, respectively. Observe also, for example, that $\sigma-\tau$ and $\sigma-1$ generate isomorphic WMAs by preceding remarks.

We return to this example in Corollary 4.2 and in Section 5 .

2. The Haar WMA over $F$ : If $L$ is the principal left ideal of $F G$ generated by $\sigma-(\tau+1)$, then $V=F G / L$ is called the Haar weak multiresolution analysis. If $v_{0}=1+L$ is the scaling function and $v_{-1}=\sigma^{-1} v_{0}$, then the scaling equation for $V$ is $v_{0}=v_{-1}+\tau_{-1} v_{-1}$. This module is usually defined over $\mathbb{R}$ but is now seen to have an analog over any field. We shall return to this example in Section 5.

It is an exercise to see that if $F=\mathbb{R}$ and $V$ is the classical multiresolution analysis with $v_{0}=\chi_{[0,1)}$ the characteristic function of the unit interval (with $V \subseteq L^{2}(\mathbb{R})$ ), then $V$ is not finitely generated over $\mathbb{R} B$.

\section{Structure OF the Group RING $F G$}

Proposition 2.1(5) suggests that one method for studying WMAs is to determine the quotients of the group ring $F G$ by certain left ideals. We take this approach by first viewing this group ring as a twisted polynomial ring with coefficients from a Bezout domain 1 One advantage of this formalism is that it suggests analogies with other important fields of study such as Drinfeld modules (additive polynomials) and differential algebra (see [10]). This sort of observation begins to place the study of MRA within a commutative algebra and algebraic geometry context.

\footnotetext{
${ }^{1} \mathrm{~A}$ Bezout domain is an integral domain in which every finitely generated ideal is principal.
} 
Since $B_{0} \cong \mathbb{Z}$, the group ring $F G$ is isomorphic to the $\operatorname{ring} F\left[T, T^{-1}\right]$ of polynomials in the variable $T$ and its inverse, where $\tau^{i} \mapsto T^{i}$ for $i \in \mathbb{Z}$. This ring is the localization of the polynomial ring $F[T]$ at the set of nonnegative powers of $T$, hence by elementary means or standard results on localization is a principal ideal domain ([7, Section 15.4]). In particular, every nonzero ideal is generated by a unique, monic polynomial $p(T) \in F[T]$ with $p(0) \neq 0$; and $F\left[T, T^{-1}\right] /(p(T)) \cong F[T] /(p(T))$ is vector space over $F$ of dimension $\operatorname{deg} p$. Each group $\operatorname{ring} F B_{j}$ is likewise isomorphic to the P.I.D. $R_{j}=F\left[T^{2^{j}}, T^{-2^{j}}\right]$ for $j \in \mathbb{Z}$, where $\tau_{j}=\sigma^{j} \tau \sigma^{-j} \mapsto T^{2^{j}}$. The nesting $B_{j} \subseteq B_{j-1}$ is reflected in the relation $R_{j} \subseteq R_{j-1}$, and

$$
R=\bigcup_{j=0}^{-\infty} F\left[T^{2^{j}}, T^{-2^{j}}\right] \cong F B
$$

Since $R$ is an increasing union of P.I.D.s, it is a Bezout domain. Finally, since $F G$ is a free left module over its subring $F B$ with basis the integer powers of $\sigma$, we may define a "twisted polynomial ring" over $R$ in the variables $S$ and $S^{-1}$ as follows. Let $R\left\{S, S^{-1}\right\}$ be the ring whose elements are uniquely expressed as formal sums:

$$
R\left\{S, S^{-1}\right\}=\left\{\sum_{i=-N}^{M} r_{i}(T) S^{i} \mid r_{i}(T) \in R, M, N \in \mathbb{Z}^{\geq 0}\right\},
$$

with addition by adding coefficients of like powers of $S$, and multiplication given by the relation $\sigma \tau=\tau^{2} \sigma$ :

$$
\left(\sum_{i=-N}^{M} a_{i}(T) S^{i}\right)\left(\sum_{j=-P}^{Q} b_{j}(T) S^{j}\right)=\sum_{k=-N-P}^{M+Q} \sum_{i+j=k} a_{i}(T) b_{j}\left(T^{2^{i}}\right) S^{k},
$$

where $b_{j}\left(T^{2^{i}}\right)$ indicates replacing each power $T^{2^{t}}$ in the expression for $b_{j}(T) \in R$ by $T^{2^{t+i}}$, for all $i, t \in \mathbb{Z}$. These operations in $R\left\{S, S^{-1}\right\}$ give a well-defined ring structure since they are simply a transport of the ring structure of $F G$ to this "polynomial" setting. Note that the units in $R$ are the union of the units in the $R_{j}$ for $j \in \mathbb{Z}$, hence are of the form $a T^{b}$ for some $a \in F-\{0\}$ and $b \in \mathbb{Z}[1 / 2]$.

Under this isomorphism between $F G$ and $R\left\{S, S^{-1}\right\}$ each cyclic $F G$-module with generator $v_{0}$ is isomorphic to $R\left\{S, S^{-1}\right\} / \mathcal{L}$, where $\mathcal{L}$ is a left ideal of $R\left\{S, S^{-1}\right\}$ and $v_{0} \mapsto 1+\mathcal{L}$. By Proposition 2.1(5), $V$ is a WMA of level $j$ when $\mathcal{L}$ contains $S-g$, for some $g \in R_{j}$. In the latter case, $V_{j, i} \cong R_{j+i} /\left(R_{j+i} \cap \mathcal{L}\right)$.

Note that there is some latitude in the specification of a ring isomorphism from $F G$ to $R\left\{S, S^{-1}\right\}$ : it is possible to map $(\sigma, \tau)$ to $\left(T^{-b} S, T\right)$ or map $(\sigma, \tau)$ to $\left(S, T^{2^{i}}\right)$, for any $b \in \mathbb{Z}[1 / 2]$ or $i \in \mathbb{Z}$, since these mappings correspond to following the originally specified isomorphism by conjugation in $R\left\{S, S^{-1}\right\}$ by the units $T^{b}$ and $S^{i}$, respectively. For conjugations of the first type, if the ideal $\mathcal{L}$ contains $S-g$ for $g \in R_{j}$, then the conjugate ideal $T^{b} \mathcal{L} T^{-b}$ also contains $T^{-b} S-g$ and hence contains $S-T^{b} g$; and if $T^{b} \in R_{j}$, then this conjugation preserves the level $j$ of the WMA. Conjugation by $S^{i}$ maps $S-g$ to $S-S^{i} g S^{-i}$; so if $g \in R_{j}$, then $S^{i} g S^{-i} \in R_{j+i}$. Thus conjugation by $S^{i}$ maps WMAs of level $j$ to ones of level $j+i$. These conjugations are simply translating and rescaling, as remarked upon previously.

The next result is a special case of a "left division algorithm" in $R\left\{S, S^{-1}\right\}$. 
Proposition 3.1. Let $F=S-g \in R\left\{S, S^{-1}\right\}$ for some $g \in R$. Then for any $H \in R\{S\}$ there is some $Q \in R\{S\}$ such that

$$
H=Q F+r, \quad \text { for some } r \in R .
$$

Proof. Since $S-g$ is monic in the variable $S$, the proof of this result is the same as that of the usual Division Algorithm [7, Theorem 3, Section 9.2].

Proposition 3.2. If $\mathcal{L}$ is a left ideal of $R\left\{S, S^{-1}\right\}$ that contains $F=S-g$, for some $g \in R$, then either $\mathcal{L}$ is the principal left ideal $R\left\{S, S^{-1}\right\} F$ or $\mathcal{L} \cap R \neq 0$.

Proof. If $\mathcal{L}$ is not the principal left ideal generated by $S-g$, then there is some $H \in \mathcal{L}$ that is not a left multiple of $F$. Multiplying $H$ on the left by a power of $S$, we may assume $H \in R\{S\}$. By the preceding result, $H=Q F+r$ for some $r \in R$. Thus $r=H-Q F$ is a nonzero element of $\mathcal{L} \cap R$, as needed to complete the proof.

\section{Characterizations of WMAs, submodules, and Quotient modules}

Continuing the notation from the previous section, $V$ is a cyclic $F G$-module with generator $v_{0}$. We initially focus on when $V$ is infinite-dimensional. By Proposition 7.1 in Section 7, such results apply to a classical multiresolution analysis over $\mathbb{R}$.

Theorem 4.1. Let $V$ be a WMA over $F$ with scaling function $v_{0}$ and, as in Proposition 2.1(5), let $L$ be the left ideal of $F G$ annihilating $v_{0}$. The following are equivalent:

(1) $V$ is infinite dimensional over $F$.

(2) $L$ is the principal left ideal generated by $\sigma-\beta$, for some nonzero $\beta \in F B$.

(3) $L \cap F B=0$.

(4) There is a unique $\beta \in F B_{0}$ such that $\sigma v_{0}=\beta v_{0}$ (i.e., the scaling equation for $v_{0}$ is unique).

In particular, an infinite-dimensional WMA over any field is determined by its scaling equation.

Proof. (3) $\Rightarrow(2)$ : This is Proposition 3.2 interpreted in $F G$ by our identification with $R\left\{S, S^{-1}\right\}$.

$(2) \Rightarrow(3)$ : Again working in $R\left\{S, S^{-1}\right\}$, assume $\mathcal{L}$ is the principal left ideal generated by $S-g$, for some nonzero $g \in R$. If $p(S)(S-g) \in \mathcal{L} \cap R$ for some $p(S) \in R\left\{S, S^{-1}\right\}$, then by examining the highest power $S$ in the product-which is obtained by multiplying the nonzero term in $p$ having the highest power of $S$, times $S$-we see that all powers of $S$ in $p(S)$ must be negative. Likewise, the lowest power of $S$ in the product is the lowest nonzero term in $p$ times $g$, and so $p$ can have no nonzero negative terms, i.e., $p=0$ as desired.

$(3) \Rightarrow(1)$ : If (3) holds, then the infinite-dimensional space $F B$ maps injectively into $F G / L \cong V$. This gives (1).

$(1) \Rightarrow(3)$ : Let $V$ be a WMA of level $j$, and let $V_{i}=V_{j, i-j}$ for all $i$. Working again with $V \cong R\left\{S, S^{-1}\right\} / \mathcal{L}$, assume $R \cap \mathcal{L} \neq 0$. Then for some $i \in \mathbb{Z}, \mathcal{L} \cap R_{i}$ is a nonzero ideal in $R_{i}$, hence has finite codimension in $R_{i}$ by a previous observation. Thus $V_{i} \cong R_{i} /\left(\mathcal{L} \cap R_{i}\right)$ is finite dimensional over $F$. Since $\sigma^{-1}$ is an isomorphism from $V_{i}$ onto $V_{i-1}$ and $V_{i} \subseteq V_{i-1}$ we obtain $V_{i}=V_{i-1}$. By applying powers of $\sigma$ we then get that $V_{i}=V_{k}$ for all $i, k$, and hence $V=V_{i}$ is finite dimensional. Thus if (1) holds, we must have $R \cap \mathcal{L}=0$, which is (3). 
$(3) \Leftrightarrow(4)$ : The scaling function $v_{0}$ satisfies scaling equations $\sigma v_{0}=\beta_{i} v_{0}$ for $i=1,2$ if and only if $\beta_{1}-\beta_{2} \in F B \cap L$. This gives the equivalence of (3) and (4).

The final remark is a recapitulation of the result that when the dimension of $V$ is infinite, its isomorphism type is uniquely determined by its scaling equation by (2) and Proposition 2.1(5).

Corollary 4.2. If $V$ is an infinite-dimensional WMA that is finitely generated over $F B$, then $V$ is isomorphic to a principal WMA.

Proof. Assume $V$ is an infinite-dimensional WMA that is finitely generated over $F B$, hence $V=F B v_{0}$ for some $v_{0} \in V$ by Corollary 2.3. Then $\sigma^{-1} v_{0}=\beta v_{0}$ for some $\beta \in F B$. By the same reasoning as the Remarks on translating and rescaling in Section 2, we may assume $v_{0}$ is chosen with $\sigma^{-1} v_{0}=f(\tau) v_{0}$ for some $f(X) \in F[X]$ with $f(0) \neq 0$. Thus $v_{0}=f\left(\tau^{2}\right) \sigma v_{0}$. By Theorem 4.1(3), $F B_{0} v_{0}$ is free of rank 1. Since $f\left(\tau^{2}\right) v_{0}$ is a generator for this module, $f\left(\tau^{2}\right)$ must be a unit in $F B_{0} \cong F\left[X, X^{-1}\right]$. It follows that $f(X)=c$ for some $c \in F^{\times}$. Thus $V$ has a scaling function $v_{0}$ with scaling equation $v_{0}=\sigma^{-1} c^{-1} v_{0}$, and so $V$ is isomorphic to a principal WMA by Theorem 4.1, as claimed.

Theorem 4.3. Let $V$ be an infinite-dimensional WMA of level zero with respect to $v_{0}$ with $v_{0}=\sigma^{-1} f(\tau) v_{0}$ for some $f(X) \in F[X]$. Let $W$ be any proper, nonzero $F G$-submodule of $V$. Then there are polynomials $p(X), q(X) \in F[X]$ such that the following hold:

(1) $p(X)$ is monic with nonzero constant term, $p\left(X^{2}\right)=q(X) p(X)$, and the roots of $p(X)$ are $\left(2^{m}-1\right)^{\text {st }}$ roots of unity, for some $m \in \mathbb{Z}^{+}$.

(2) $p(X)$ and $f(X)$ are relatively prime.

(3) The submodule $W$ is a WMA of level zero with scaling function $w_{0}=p(\tau) v_{0}$ and scaling equation $w_{0}=\sigma^{-1} q(\tau) f(\tau) w_{0}$.

(4) The quotient module $\bar{V}=V / W$ is a WMA of level zero and finite dimension with $\bar{V}=\overline{V_{0, i}}$ for all $i \in \mathbb{Z}$.

(5) The minimal polynomial of $\tau^{2^{i}}$ acting on $\bar{V}$ is $p(x)$, for all $i \in \mathbb{Z}$.

Proof. Let $V_{i}=V_{0, i}$ for all $i$. Let $W$ be any nonzero, proper $F G$-submodule of $V$, and let overbars denote the natural projection of $V$ onto $V / W$. By Corollary 2.4, $\bar{V}$ is a WMA of level zero with respect to $\overline{v_{0}}$. Let $L$ be the annihilator in $F G$ of the scaling function $v_{0}$, and let $L_{1}$ be the left ideal of $F G$ which is the preimage of $W$ under the presentation homomorphism $\Phi$ in Proposition 2.1(5). Let $\mathcal{L}$ and $\mathcal{L}_{1}$ be the ideals in $R\left\{S, S^{-1}\right\}$ corresponding to $L, L_{1}$, respectively.

Since $\mathcal{L} \subset \mathcal{L}_{1}$, by Proposition 3.2, $\mathcal{L}_{1} \cap R \neq 0$. As in the proof of Theorem 4.1, there is a positive integer $n$, independent of $i$, such that $R_{i} \cap \mathcal{L}_{1}$ has codimension $n$ in $R_{i}$ for all $i$. Moreover,

$$
\overline{V_{i}} \cong R_{i} /\left(R_{i} \cap \mathcal{L}_{1}\right) \text { has dimension } n \text { over } F \text {, for all } i \in \mathbb{Z} .
$$

Again as in Theorem 4.1, since $\sigma^{-1}$ maps $\overline{V_{i}}$ onto the subspace $\overline{V_{i-1}}$ containing it,

$$
\bar{V}=\overline{V_{i}} \text { has dimension } n \text { over } F \text {. }
$$

Since $R_{i}$ is a principal ideal domain, there is a unique, monic polynomial $p_{i}$ in the variable $T^{2^{i}}$ with $p_{i}(0) \neq 0$ such that $\overline{V_{i}} \cong F\left[T^{2^{i}}\right] /\left(p_{i}\right)$; moreover, $\left(p_{i}\right)$ is the minimal polynomial of $\tau_{i}$ acting on $\overline{V_{i}}=\bar{V}$, and the degree of $p_{i}$ in its variable $T^{2^{i}}$ is $n$. Since $\tau_{i}$ is conjugate to $\tau_{i+1}$ in $G$, their minimal polynomials are the same: $p_{i}(X)=p_{i+1}(X)$ for all $i$, where $X$ is an independent variable. 
Let $p(X)=p_{i}(X)$. Since $\tau$ is conjugate to $\tau^{2^{i}}, p(X)$ divides $p\left(X^{2^{i}}\right)$ in $F[X]$, for all $i \in \mathbb{Z}^{+}$. It follows easily that for some $m$ dividing $n$ !, each root, $\lambda$, of $p$ (in an algebraic closure of $F$ ) satisfies $\lambda^{2^{m}}=\lambda$. Thus the roots of $p$ are $\left(2^{m}-1\right)^{\text {st }}$ roots of unity.

We next show that $p(X)$ and $f(X)$ are relatively prime. If $d(X)$ is their g.c.d., then $\sigma \overline{v_{0}}=f(\tau) \overline{v_{0}} \subseteq d(\tau) \bar{V}$. Note that $d(\tau) \bar{V}$ is a subspace of $\bar{V}$ stable under $\tau$ and annihilated by a polynomial in $\tau$ which is a divisor of $p$. Since $p$ is also the smallest degree polynomial in $\tau$ that annihilates $\sigma \overline{v_{0}}$, we must have $d(\tau) \bar{V}=\bar{V}$ and so $d=1$ as claimed.

Finally, we show that $W$ is a WMA. Let $w_{0}=p(\tau) v_{0}$, so that $\overline{w_{0}}=\overline{0}$, i.e., $w_{0} \in W$. Let $W^{\prime}=F G w_{0}$. By the preceding discussion $W^{\prime}$ has finite codimension $n^{\prime}$ in $V$, where $n^{\prime}$ equals the codimension of $W^{\prime} \cap V_{0}$ in $V_{0}$. By construction, however, the codimension of $F B_{0} w_{0}$ in $V_{0}$ equals $\operatorname{deg} p$. Thus $n^{\prime} \leq \operatorname{deg} p=\operatorname{codim} W$. Since $W^{\prime}$ is a submodule of $W$, we must have $W^{\prime}=W$, i.e., $w_{0}$ is a cyclic generator for $W$. To find the scaling equation for $w_{0}$ let $p\left(X^{2}\right)=q(X) p(X)$ in $F[X]$. Then

$$
\sigma w_{0}=\sigma p(\tau) v_{0}=p\left(\tau^{2}\right) \sigma v_{0}=p\left(\tau^{2}\right) f(\tau) v_{0}=q(\tau) p(\tau) f(\tau) v_{0}=q(\tau) f(\tau) w_{0} .
$$

This establishes (3), and completes all parts of the proof.

To complete the picture we now give some characterizations of finite-dimensional WMAs.

Theorem 4.4. Let $V$ be a finite-dimensional $W M A$ of level zero over a field $F$ with char $F \neq 2$. Let $p(X) \in F[X]$ be the minimal polynomial of $\tau$ acting on $V$, and assume also that $F$ contains all roots of $p$. Then the following hold:

(1) $V=V_{0, i}$ for all $i \in \mathbb{Z}$.

(2) $p(X)=p_{1}(X) \cdots p_{r}(X)$, where for each $i \in\{1, \ldots, r\}$ there is a root $\lambda_{i}$ of $p_{i}(X)$ such that $\lambda_{i}, \lambda_{i}^{2}, \ldots, \lambda_{i}^{2^{m_{i}-1}}$ are all the distinct roots of $p_{i}(X)$, and $\lambda_{i}^{2^{m_{i}}}=\lambda_{i}$.

(3) $V=V_{1} \oplus V_{2} \oplus \cdots \oplus V_{r}$ is a decomposition of $V$ into $F G$-submodules, where $V_{i}$ is annihilated by $p_{i}(\tau)$.

(4) Let $\operatorname{dim} V_{i}=e_{i} m_{i}$ and let $J_{i}$ be the $e_{i} \times e_{i}$ elementary (lower triangular) Jordan block with eigenvalue $\lambda_{i}$. Then there is a basis of $V_{i}$ such that $\tau$ and $\sigma$ acting on $V_{i}$ with respect to this basis have matrices

$$
[\tau]=\left(\begin{array}{cccc}
J_{i} & & & 0 \\
& J_{i}^{2} & & \\
& & \ddots & \\
0 & & & J_{i}^{2^{m_{i}-1}}
\end{array}\right) \text { and }\left[\sigma^{-1}\right]=\left(\begin{array}{ccccc}
0 & 0 & \ldots & 0 & A \\
I & 0 & \ldots & \ldots & 0 \\
0 & I & 0 & \ldots & \vdots \\
\vdots & & \ddots & & \vdots \\
0 & \ldots & \ldots & I & 0
\end{array}\right) \text {, }
$$

where $I$ is the $e_{i} \times e_{i}$ identity matrix, and $A$ is an $e_{i} \times e_{i}$ matrix that is uniquely (and explicitly) determined by the scaling equation for $V$ and the polynomial $p$.

Proof. As usual, we may assume the scaling equation for $V$ is $v_{0}=\sigma^{-1} f(\tau) v_{0}$ for some $f(X) \in F[X]$. Since $V$ is a homomorphic image of the WMA $F G / L$, where $L$ is the principal left ideal generated by $\sigma-f(\tau)$, we may apply Theorem 4.3 to obtain parts (1) and (2), where $p_{i}$ is the product of all factors whose roots lie in an orbit of the group of 2-power maps acting on the roots of $p$. 
Next note that if $\lambda$ is any root of $p$, then $\lambda^{2^{m}-1}=1$ for some $m$. Thus since $F$ has odd characteristic, $-\lambda$ is not a root of $p$, i.e., $\lambda^{2^{m-1}}$ is the unique square root of $\lambda$ that is a root of $p$. Thus for any positive integer $k$ we have $\left(\tau^{2}-\lambda^{2}\right)^{k} v=0$ if and only if $(\tau-\lambda)^{k} v=0$. To obtain (3) note that if $(\tau-\lambda)^{k} v=0$ for some $v \in V$, then

$$
0=(\tau-\lambda)^{k} v=\left(\tau^{2}-\lambda^{2}\right)^{k} v=\left(\sigma\left(\tau-\lambda^{2}\right)^{k} \sigma^{-1}\right) v=\left(\tau-\lambda^{2}\right)^{k}\left(\sigma^{-1} v\right) .
$$

Thus if $V_{\lambda}$ is the $(X-\lambda)$-primary component of $\tau$ acting on $V$, then $\sigma^{-1} V_{\lambda}=V_{\lambda^{2}}$. In particular, $\langle\sigma\rangle$ permutes the primary components associated to roots that are 2-powers of each other, and so (3) follows.

To establish (4) we may simplify notation by assuming $V=V_{1}$ and the distinct roots of $p$ are $\lambda, \lambda^{2}, \ldots, \lambda^{2^{m-1}}$. Also let $V=U_{0} \oplus \cdots \oplus U_{m-1}$, where $U_{i}=V_{\lambda^{2^{i}}}$, and $\sigma^{-1} U_{i}=U_{i+1}$ with the indices read $\bmod n$. Note that

$$
p(X)=\prod_{i=0}^{m-1}\left(X-\lambda^{2^{i}}\right)^{e} \quad \text { where } e=\operatorname{dim} U_{i} \text { for all } i .
$$

Let $\mathcal{B}$ be an ordered basis of $U_{0}$ for which the matrix, $J$, of $\tau$ is the $e \times e$ lower triangular Jordan block with eigenvalue $\lambda$. For each $i=1,2, \ldots, m-1$ let $\mathcal{B}_{i}=\sigma^{-i} \mathcal{B}$ (with the corresponding basis ordering). It follows that $\mathcal{B}_{i}$ is a basis of $U_{i}$ and the matrix of $\tau$ on $U_{i}$ with respect to $\mathcal{B}_{i}$ is $J^{2^{i}}$. Let $A$ be the $e \times e$ matrix representing the action of $\sigma^{2^{m}}$ on $U_{0}$ with respect to $\mathcal{B}$. All parts of the theorem now follow once we have established the uniqueness of the matrix $A$ in (4.1).

By construction, $\mathcal{B}=\left\{v_{0}, v_{1}, \ldots, v_{e-1}\right\}$, where $(\tau-\lambda) v_{i}=v_{i+1}$, i.e., $\tau$ has matrix $J=J_{\lambda}$. Let $A$ be the matrix of $\sigma^{-2^{m}}$ with respect to this basis (which is the same $A$ as above). Since $A J^{2^{m}} A^{-1}=J$, the matrix $A$ is a change of basis that converts $J^{2^{m}}$ into its Jordan form on $U$. We calculate one specific such change of basis matrix, $P$, as follows. A basis for which $\tau^{2^{m}}$ is in Jordan form is $u_{0}, u_{1}, \ldots, u_{e-1}$, where $u_{i}=\left(\tau^{2^{m}}-\lambda\right)^{i} v_{0}$, for $i=0,1, \ldots, e-1$. To compute the change of basis matrix explicitly let $N=2^{m}$ and let

$$
\Phi(X)=\frac{X^{N}-\lambda^{N}}{X-\lambda}=\sum_{j=0}^{N-1} \lambda^{j} X^{N-j-1} .
$$

Then

$$
\begin{aligned}
u_{i}=\left(\tau^{N}-\lambda\right)^{i} v_{0} & =\left(\tau^{N}-\lambda^{N}\right)^{i} v_{0} \\
& =\Phi(\tau)^{i}(\tau-\lambda)^{i} v_{0}=\Phi(\tau)^{i} v_{i}
\end{aligned}
$$

Thus, writing $\Phi(\tau)^{i} v_{i}$ in terms of the basis $\mathcal{B}$ gives the $i^{\text {th }}$ column of the matrix $P^{-1}$.

Finally, to establish the uniqueness in part (4), if $A$ is the matrix that represents $\sigma^{-2^{m}}$ with respect to $\mathcal{B}$, then $A^{-1} J A=J^{2^{m}}=P^{-1} J P$. Thus $P A^{-1}=C$ commutes with $J$. Now by repeated iterations of the scaling equation we obtain

$$
\sigma^{2^{m}} v_{0}=f_{1}(\tau) v_{0} \quad \text { for some } f_{1}(X) \in F[X]
$$

where $f_{1}$ is uniquely determined by $f$ and the power $2^{m}$ (which is determined by $p$ ). Since the scaling function $v_{0}$ for $V$ projects onto a cyclic generator of $U_{0}$ - which was arbitrary and also denoted by $v_{0}$-we have

$$
C v_{0}=P A^{-1} v_{0}=P \sigma^{2^{m}} v_{0}=P f_{1}(\tau) v_{0} .
$$


Thus $C$ is uniquely determined on $v_{0}$ by the explicit matrix $P$, the scaling polynomial $f$ and the power $2^{m}$. Since $C$ commutes with $(\tau-\lambda)^{i}$ for all $i$, the matrix of $C$ with respect to the fixed basis $\mathcal{B}$ is uniquely determined on $U_{0}$, hence so is $A$, as needed.

Observe that by choosing $A$ as the explicitly computed matrix $P$ above, the matrices in (4.1) determine a representation of $G$ on the vector space $V$. However, this representation need not satisfy a given scaling equation (hence the introduction of the matrix $C$ in (4.3) to highlight the latitude in choices for $A$ ). It is also easily seen that $C$ is necessarily a polynomial in $J$ of degree at most $e-1$.

From (4.2) we may compute the $i, i$ diagonal entry of the matrix $P$ to be $\Phi(\lambda)^{i-1}=2^{m(i-1)}$. In particular, if these powers of 2 are distinct in $F$, then $\sigma^{2^{m}}$ is similar to a diagonal matrix over $F$ (with respect to a different basis, not necessarily preserving the "Jordan form" of $\tau$ ).

A special case of Theorem 4.4 is worth recording:

Corollary 4.5. In the notation of Theorem 4.4, if $p(X)=(X-1)^{e}$, then a representation for $G$ is obtained by mapping $\tau$ to the $e \times e$ Jordan block $J$ with eigenvalue 1 , and mapping $\sigma$ to $P^{-1}=\left(a_{i, j}\right)$, where

$$
a_{i, j}= \begin{cases}0, & \text { if } i<j, \\
2^{2 j-i-1}\left(\begin{array}{l}
j-1 \\
i-j
\end{array}\right), & \text { if } i \geq j .\end{cases}
$$

Up to equivalence, all representations are then obtained by replacing $P$ by $P C$, where $C$ is invertible and a polynomial in $J$ of degree at most $e-1$.

Proof. In this case $r=1, m_{1}=1$ and $\left(a_{i, j}\right)$ is the matrix $P^{-1}$ computed from (4.2) with $\Phi(X)=X+\lambda$. The coefficients are thus obtained directly from the binomial expansion.

\section{WAVELETS}

In this section $V$ is an infinite-dimensional WMA with scaling function $v_{0}$. By the Remarks following Corollary 2.4 we may assume $V$ is of level zero and the scaling equation is $v_{0}=\sigma^{-1} f(\tau) v_{0}$, for some polynomial $f(X) \in F[X]$. For each integer $i$ let $V_{i}=V_{0, i}$. It follows from Theorem 4.1(3) that $V_{-1}=\sigma^{-1} V_{0}$ is a free $F B_{-1}$-module of rank 1 , and a free $F B_{0}$-module of rank 2 (since $B_{0}$ is of index 2 in $\left.B_{-1}\right)$. Moreover, if we let

$$
v_{-1}=\sigma^{-1} v_{0} \quad \text { and } \quad v_{-1}^{*}=\tau_{-1} v_{-1},
$$

then $\left\{v_{-1}\right\}$ is an $F B_{-1}$-basis and $\left\{v_{-1}, v_{-1}^{*}\right\}$ is an $F B_{0}$-basis for $V_{-1}$.

Definition. Let $V$ be an infinite-dimensional WMA of level zero with respect to the scaling function $v_{0}$. Say $V$ has a wavelet basis with respect to $v_{0}$ if the $F B_{0^{-}}$ submodule $F B_{0} v_{0}$ is a direct summand (or pure submodule) of $V_{-1}$ considered as an $F B_{0}$-module. If $V$ has such a wavelet basis, then a mother wavelet is any element in $V_{-1}$ that generates an $F B_{0}$-module direct sum complement to $F B_{0} v_{0}$ in $V_{-1}$.

The importance of a wavelet basis is in obtaining successively "finer" resolutions, as follows. If $V_{0}$ is a direct summand of $V_{-1}$ considered as an $F B_{0}$-module, then for all $i \geq 0, V_{-i}$ is likewise an $F B_{-i}$-module direct summand of $V_{-i-1}$, and so 
$V_{-i-1}=V_{-i} \oplus W_{-i}$, for some cyclic $F B_{-i}$-module $W_{-i}$. Thus we obtain for all positive $i$ a decomposition

$$
V_{-i}=V_{-i+1} \oplus W_{-i+1}=V_{0} \oplus W_{0} \oplus W_{-1} \oplus W_{-2} \oplus \cdots \oplus W_{-i+1},
$$

where the $W_{-j}$ are free $F B_{0}$-modules with bases given by sets of scaled and translated versions of the "mother wavelet" generating $W_{0}$ (cf. the Introduction). The $V_{-i+1}$ component of a vector in $V_{-i}$ may be thought of as an approximation to a vector (or function), and the "error" in this approximation is its component in $W_{-i+1}$. Successively refining this process as in (5.1) is integral to the efficacy of a multiresolution analysis. Note that this process cannot be carried through $V_{i}$ for $i>0$ for want of an $F B_{0}$-module structure (although the arbitrary choice of "level zero" in the resolution can be tailored to the degree of detail or resolution needed in a given application).

A classical multiresolution analysis over $\mathbb{R}$ (assuming the $V_{i}$ are closed subspaces) always has a wavelet basis since the submodule $F B_{0} v_{0}$ has an orthogonal complement in $V_{-1}$ that is also an $F B_{0}$-submodule. Since orthogonality is not generally available for WMAs over $F$, not every WMA need have a wavelet basis with respect to a given generator. We shall see, however, that every WMA has a wavelet basis with respect to some generator.

Theorem 5.1. Let $V$ be an infinite WMA of level zero with scaling function $v_{0}$ whose scaling equation is a polynomial in $\tau$. Write the scaling equation as

$$
v_{0}=\sigma^{-1}\left(\tau^{2 n_{1}} \tau f_{1}\left(\tau^{2}\right)+\tau^{2 n_{2}} f_{2}\left(\tau^{2}\right)\right) v_{0}, \quad f_{1}(T), f_{2}(T) \in F[T], n_{1}, n_{2} \in \mathbb{Z}^{\geq 0},
$$

where each of $f_{1}(T)$ and $f_{2}(T)$ is either zero or has nonzero constant term. Then $V$ has a wavelet basis with respect to $v_{0}$ if and only if one of the following holds:

(1) Exactly one of $f_{1}$ or $f_{2}$ is zero and the other is a nonzero constant.

(2) Both $f_{1}$ and $f_{2}$ are nonzero and they are relatively prime in $F[T]$.

Proof. By collecting the odd and even power monomial terms of $\tau$, and factoring out integer powers of $\tau$, the scaling equation may always be written in the form described; and $f_{1}, f_{2}$ cannot both be zero. We may then rewrite the scaling equation as

$$
v_{0}=\left(\tau^{n_{1}} \tau_{-1} f_{1}(\tau)+\tau^{n_{2}} f_{2}(\tau)\right) \sigma^{-1} v_{0}=\tau^{n_{1}} f_{1}(\tau) v_{-1}^{*}+\tau^{n_{2}} f_{2}(\tau) v_{-1}
$$

By observations preceding this theorem, this is the unique expression of $v_{0}$ with respect to the $F B_{0}$-basis elements $v_{-1}^{*}$ and $v_{-1}$. Since $V_{-1}$ is a module over the Principal Ideal Domain $F B_{0} \cong F\left[T, T^{-1}\right]$, by [7, Theorem 12.4] a nonzero element $w$ generates a direct summand if and only if $w$ is not of the form $g v$ for any nonunit $g \in F B_{0}$ and any $v \in V_{-1}$. Equivalently, $w$ generates a direct summand if and only if its coefficients, when expressed in terms of a basis, have no common nonunit divisor. Since the powers of $T$ are units in $F\left[T, T^{-1}\right]$, after removing these as we did to normalize the basis coefficients, the theorem now follows immediately from the fact that the g.c.d. of two polynomials in $F[T]$ is the same (up to units) as their g.c.d. in $F\left[T, T^{-1}\right]$.

Corollary 5.2. Let $V$ be a WMA with respect to $v_{0}$ with scaling equation $v_{0}=$ $\sigma^{-1} f(\tau) v_{0}$ for $f(X) \in F[X]$. Then $V$ has a wavelet basis with respect to $v_{0}$ if and only if we cannot write $f(X)=g\left(X^{2}\right) h(X)$ for some $g(X), h(X) \in F[X]$ with $g$ not a constant times a power of $X$.

Proof. This is immediate from Theorem 5.1. 
Corollary 5.3. In the notation of Theorem 5.1, $V$ has a wavelet basis with respect to $v_{0}$ if and only if

$$
1=a(T) f_{1}(T)+b(T) f_{2}(T), \quad \text { for some } a(T), b(T) \in F[T] .
$$

In this case the element $b(\tau) v_{-1}^{*}-a(\tau) v_{-1}$ is a mother wavelet.

Proof. The first assertion is the familiar property of g.c.d.s in a P.I.D. When this condition holds, let $w_{1}=v_{0}$ and $w_{2}=b(\tau) v_{-1}^{*}-a(\tau) v_{-1}$. Then the transition matrix from the basis $\left\{v_{-1}^{*}, v_{-1}\right\}$ to the set $\left\{w_{1}, w_{2}\right\}$ is seen to have determinant a unit in $F\left[T, T^{-1}\right]$. This implies the latter set is a basis, as needed.

The condition that $f_{1}$ and $f_{2}$ be relatively prime is easily (and rapidly) checked by the Euclidean Algorithm, or by computing the resultant of $f_{1}$ and $f_{2}$; see [7] for both methods.

Corollary 5.4. Assume $V$ has a wavelet basis with respect to $v_{0}$ and, in the notation of Corollary 5.3, let $w_{2}=b(\tau) v_{-1}^{*}-a(\tau) v_{-1}$. Then $w$ is a mother wavelet for $V$ if and only if $w=\beta_{1} v_{0}+c \tau^{k} w_{2}$, for some $\beta_{1} \in F B_{0}, k \in \mathbb{Z}$, and $c \in F-\{0\}$.

Proof. This is a restatement of the observation that if $\left\{v_{0}, w_{2}\right\}$ is a basis of a free module, then $\left\{v_{0}, w\right\}$ is also a basis if and only if the $2 \times 2$ transition matrix is invertible, i.e., has unit determinant. The determinant for the specified transition equals the coefficient of $w_{2}$ when writing $w$ in terms of $\left\{v_{0}, w_{2}\right\}$. Since the units in $F\left[T, T^{-1}\right]$ are nonzero constants times integer powers of $T$, the result follows.

Definition. Let $V$ be an infinite-dimensional WMA of level $j$ with respect to the scaling function $v_{0}$ whose scaling equation is $v_{0}=\sigma^{-1} f\left(\tau_{j}\right) v_{0}$ for some $f(X) \in$ $F\left[X, X^{-1}\right]$. We say the scaling function or scaling equation is reduced if $f(X) \in$ $F[X], f(0) \neq 0$, and we cannot write $f(X)=g\left(X^{2}\right) h(X)$ for some $g(X), h(X) \in$ $F[X]$ with $g$ not a constant times a power of $X$.

We also say the scaling polynomial $f(X)$ is reduced if the corresponding equation is reduced. By the Remarks following Corollary 2.4, every scaling function may be replaced by a translated scaling function whose scaling equation is a polynomial with nonzero constant term. Furthermore, $V$ may be rescaled so the scaling function is of level zero, with the same scaling polynomial (so we lose no generality in assuming the scaling function is of level zero). By Corollary 5.2, reduced scaling functions are those for which $V$ has a wavelet basis.

Theorem 5.5. Let $V$ be an infinite-dimensional WMA.

(1) Then $V$ has a reduced scaling function of level zero, hence has wavelet basis with respect to that scaling function. More specifically, over all scaling functions of level zero, if $v_{0}$ is one of minimal degree, then $v_{0}$ is reduced.

(2) Any two reduced scaling functions in $V$ have the same scaling equation.

Proof. (1): Choose a scaling function $v_{0}$ of level zero so that its scaling equation is $v_{0}=\sigma^{-1} f(\tau) v_{0}$ with $f(X) \in F[X]$ of minimal degree. If $v_{0}$ is not reduced, we may write

$$
f(X)=g\left(X^{2}\right) h(X), \quad \text { for some } g, h \in F[X] \text { with } g(X) \neq c X^{n} .
$$

Let $w_{0}=h\left(\tau_{-1}\right) v_{-1}$. Since $v_{0}=g(\tau) h\left(\tau_{-1}\right) \sigma^{-1} v_{0}=g(\tau) w_{0} \in F G w_{0}$ we have that $w_{0}$ is a cyclic generator for $V$. Now

$$
\sigma w_{0}=\sigma h\left(\tau_{-1}\right) v_{-1}=h(\tau) \sigma v_{-1}=h(\tau) v_{0}=h(\tau) g(\tau) w_{0}
$$


so $w_{0}$ is a scaling function of level zero and of smaller degree than $v_{0}$, a contradiction. This establishes (1).

(2): As in the Remarks following Corollary 2.4, by replacing the given scaling functions by suitable $\sigma$-powers times them (which does not alter their scaling equations) we may assume $v_{0}$ and $u_{0}$ are reduced scaling functions of level zero. Now there is some smallest nonnegative integer $k$ such that $v_{0} \in U_{-k}$, where, as usual, $U_{i}=F B_{i} u_{i}$, where $u_{i}=\sigma^{i} u_{0}$. Since $U_{-k}$ is an $F B_{0}$-module, $V_{0} \subseteq U_{-k}$. Likewise there is some $m \geq k$ such that $u_{-k} \in V_{-m}$, and hence $U_{-k} \subseteq V_{-m}$. Considering all modules over $F B_{0}$, since $v_{0}$ is a reduced scaling function, by (5.1) $V_{0}$ is a direct summand of $V_{-m}$, hence is a direct summand of the submodule $U_{-k}$ of $V_{-m}$ containing it. Likewise $U_{0}$ is a direct summand of $U_{-k}$. Since $V_{-m}$ is a free module over the P.I.D. $F B_{0}$, either $U_{0}=V_{0}$ or $U_{0} \cap V_{0}=0$.

If $U_{0}=V_{0}$, then $u_{0}=\alpha v_{0}$ for some unit $\alpha \in F B_{0}$. In this case $\alpha=c \tau^{i}$ for some $c \in F^{\times}$and $i \in \mathbb{Z}$. Since the scaling equations for $u_{0}$ and $v_{0}$ are both reduced, it follows that $i=0$ and the equations are the same.

Consider when $U_{0} \cap V_{0}=0$. In this case, since $u_{0}$ is a scaling function, $\sigma^{m} u_{0} \in U_{0}$. But $\sigma^{m} u_{0} \in \sigma^{m} V_{-m}=V_{0}$, and so $\sigma^{m} u_{0} \in U_{0} \cap V_{0}=0$, a contradiction. This completes the proof.

Remarks. The method of proof of Theorem 5.5 shows how to obtain a scaling function which possesses a wavelet basis from any given scaling function: iterate the process of "removing" factors of the form $g\left(X^{2}\right)$ from the scaling equation (which clearly terminates by degree considerations). Also, each infinite-dimensional WMA $V$ has a unique reduced scaling equation (or polynomial) associated to it, namely that of any reduced scaling function in $V$. We call this the reduced scaling equation for $V$. In particular, we now immediately obtain the following uniqueness result.

Corollary 5.6. Two infinite-dimensional WMAs are isomorphic as FG-modules if and only if they have the same reduced scaling equations.

Finally, if $w$ is a mother wavelet, we may write

$$
w=g_{1}(\tau) \tau v_{-1}+g_{2}(\tau) v_{-1}, \quad g_{1}(\tau), g_{2}(\tau) \in F B_{0} .
$$

This is called a wavelet equation. In the absence of orthogonality, a mother wavelet is not uniquely determined, hence the wavelet coefficients $g_{i}$ are not unique either. We also note that a mother wavelet, $w$, by the proof of Theorem 5.5(2), is not a scaling function of level zero. The following examples show that a mother wavelet may be a scaling function of level -1, or it may be a generator for $V$ as an $F G$-module but not a scaling function of any level, or indeed it need not even generate $V$ as an $F G$-module (although by Theorem 4.3 it generates a WMA of finite codimension in $V$ ).

Examples. Following the notation and results of the Examples after Corollary 2.4:

1. Principal WMAs over $\boldsymbol{F}$ : Evidently $V_{-1}=F B_{0} v_{0} \oplus F B_{0} v_{\frac{1}{2}}$. Thus $v_{\frac{1}{2}}$ is a mother wavelet. Note that $v_{\frac{1}{2}}$ is also a scaling function for $V$ of level -1 . On the other hand, if the characteristic of $F \neq 2$, then, as mentioned before, the mother wavelet $w=v_{0}+v_{\frac{1}{2}}$ is a generator for $V$ as an $F G$-module, but is not a scaling function at any level. Finally, the mother wavelet $w^{\prime}=v_{0}-v_{\frac{1}{2}}$ is not a generator for $V$ as an $F G$-module, since it lies in the image in $V$ of the augmentation ideal of $F B$ (of codimension 1 in $V$ ). 
2. The Haar WMA over $\boldsymbol{F}$ : Assume $\operatorname{char} F \neq 2$. In this example, since $v_{-1}, v_{-1}^{*}$ is an $F B_{0}$-module basis for $V_{-1}$, by Corollary $5.3 w=v_{-1}-v_{-1}^{*}$ is a mother wavelet, called the Haar wavelet over $F$. This vector does not generate $V$ as an $F G$-module because it lies in the image of the augmentation ideal of $F B$ which lies in a proper $F G$-submodule (in the classical language, it and all its scaled translates have zero mean).

Note that, for example, $v_{-1}^{*}$ is also a mother wavelet for the Haar WMA and it is a scaling function of level -1 .

3. Battle-Lemarié WMAs: These wavelets in $L^{2}(\mathbb{R})$, described in detail in 6. Section 5.4], are constructed for each $N \geq 0$ from a function $\phi$ which is a $B$ spline of degree $N$ with knots at the integers. The Haar wavelets are the degree zero Battle-Lemarié wavelets. For $N \geq 1$ the translates of $\phi$ are not orthogonal, so $\phi$ must be modified by an "orthogonalization trick" to produce a multiresolution analysis in $L^{2}(\mathbb{R})$; the scaling function that results from this process does not have compact support. Since the translates of $\phi$ are linearly independent, however, we may use $\phi$ itself to generate a weak multiresolution analysis and (algebraic) wavelet basis.

We illustrate the results of this section in the $N=2$ case. The quadratic $B$-spline $\phi$ has support $[-1,2]$ and is defined on this interval by

$$
\phi(x)=\left\{\begin{array}{lr}
\frac{1}{2}(x+1)^{2}, & -1 \leq x \leq 0, \\
\frac{3}{4}-\left(x-\frac{1}{2}\right)^{2}, & 0 \leq x \leq 1, \\
\frac{1}{2}(x-2)^{2}, & 1 \leq x \leq 2 .
\end{array}\right.
$$

The scaling equation for $\phi$ is

$$
\phi(x)=\frac{1}{4} \phi(2 x+1)+\frac{3}{4} \phi(2 x)+\frac{3}{4} \phi(2 x-1)+\frac{1}{4} \phi(2 x-2) .
$$

With $G$ acting in the usual fashion on $L^{2}(\mathbb{R})$ (see Section 1), translate $\phi$ as in the Remarks in Section 2 by setting $v_{0}=\phi(x-1)$ so that the scaling equation becomes a polynomial in $\tau$-in the notation of Theorem 5.1 it is

$$
v_{0}=\sigma^{-1}\left(\tau\left(\frac{3}{4} \tau^{0}+\frac{1}{4} \tau^{2}\right)+\left(\frac{1}{4} \tau^{0}+\frac{3}{4} \tau^{2}\right)\right) v_{0} .
$$

Hence $f_{1}(T)=\frac{3}{4}+\frac{1}{4} T$ and $f_{2}(T)=\frac{1}{4}+\frac{3}{4} T$. These polynomials are clearly relatively prime, and $\frac{3}{2} f_{1}-\frac{1}{2} f_{2}=1$. By Corollary 5.3, a mother wavelet is $-\frac{1}{2} \sigma^{-1} \tau v_{0}-$ $\frac{3}{2} \sigma^{-1} v_{0}$. Since $v_{-1}=\sigma^{-1} v_{0}$ and $v_{-1}^{*}=\sigma^{-1} \tau v_{0}$, this mother wavelet is seen to be

$$
\psi(x)=-\frac{1}{2} \phi(2 x-1)-\frac{3}{2} \phi(2 x-2) .
$$

Note that $\int_{-\infty}^{\infty} \psi(x) d x=-1$. By Corollary 5.4, the function $\psi_{1}(x)=\psi(x)+\phi(x-1)$ is another mother wavelet; it enjoys the additional property that $\psi_{1}\left(x+\frac{3}{2}\right)$ is an odd function; so, in particular, the mother wavelet $\psi_{1}$ satisfies an "admissibility" condition: $\int_{-\infty}^{\infty} \psi_{1}(x) d x=0$. Note that the scaling equation may be read in any field of characteristic not equal to 2 , thereby giving analogs of the quadratic $B$ spline wavelets in different settings.

\section{FURTHER DiRECTIONS}

One of the main purposes of this paper is to place the theory of multiresolution analyses in a larger algebraic setting, with the intent of stimulating additional theoretical and practical developments. We suggest some possible lines of further research. 
1. Generalizing to other groups. There is an obvious definition of WMAs for arbitrary integral scaling factors $N \geq 2$, obtained by replacing "2" by " $N$," and evidently the results herein carry over mutatis mutandis to such groups. An $N$-scale MRA theory for $L^{2}(\mathbb{R})$ - which falls under the general rubric of "multiwavelets" has been mentioned by others, and is developed systematically in $L^{2}(\mathbb{R})$ in $[4$. In the general algebraic setting, $N$-scale WMAs lead to the notion of an (algebraic) wavelet basis, although the direct sum complements to $V_{0}$ in $V_{-1}$ are free of rank $N-1$, so there is not a single "mother wavelet" when $N>2$ (which is the case in $L^{2}(\mathbb{R})$ as well). Examples, such as the $N$-scale analogs of the Haar wavelets, require the underlying field to contain $N$ distinct $N^{\text {th }}$ roots of unity in order to formulate mother wavelets analogous to the DFT basis functions (which are eigenfunctions for an action of a cyclic group of order $N$ ).

Generalized multiresolution analyses for other groups are explored in papers cited in the Introduction. In particular, Section VI of [9] discusses generalized multiresolution analyses for finite and profinite groups. Combining ideas from these papers with ones in this work may lead to new productive notions of WMAs for other groups, with concomitant harmonic analyses. In particular, other higherdimensional affine groups may lead to new perspectives on multi-dimensional multiresolution analyses - an area of considerable interest in signal processing.

2. $p$-adic weak multiresolution analyses. Some recent work on wavelets over local fields appears in [1] and 2. In the context of WMAs it seems natural to pass to a completion and consider $F G^{*}$-modules, where

$$
G^{*}=G^{*}(\nu)=\left\{\left(\begin{array}{cc}
\pi^{n} & b \\
0 & 1
\end{array}\right) \mid n \in \mathbb{Z}, b \in K_{\nu}\right\},
$$

and where $K_{\nu}$ is a field with a discrete valuation $\nu$ and uniformizing parameter $\pi$. Here $\pi$ would essentially play the role of 2 (or $p$ ) in the theory, and for a cyclic $F G^{*}$-module $V^{*}$ generated by $v_{0}^{*}$ let $V_{0}^{*}=F B_{0}^{*} v_{0}^{*}$, where $B_{0}^{*}$ is the unipotent subgroup of $G^{*}$ with entries from the ring of integers in $K_{\nu}$. To define a WMA in this context we might require $v_{0}^{*} \in \sigma^{-1} V_{0}^{*}$, where $\sigma=\operatorname{diag}(\pi, 1)$. In order to place the theory within the mainstream of representation theory of $p$-adic groups ([24]) it may be necessary to impose further restrictions on the representation (e.g., smoothness, admissibility, etc.), and so $V^{*}$ may need to be a projective limit of finite-dimensional modules (cf. Section 4).

Specifically for a 2-adic theory, it would be instructive to seek ties to trees and wreath product multiresolution analyses, as described in [9. More generally, the affine buildings for linear groups over $K_{\nu}$ may be brought to bear for further insight.

A coherent $p$-adic theory of WMAs may then lead naturally to an adelic formulation by taking suitable restricted direct products of $p$-adic WMAs over all primes. In both $p$-adic and adelic theories it may be instructive to revisit the classical theory of dense submodules of $L^{2}(\mathbb{R})$ in order to glean "Fourier analytic" insights whenever possible.

3. Other types of multiresolution analyses. In the notation of Section 3, it might be productive to consider $F G$-modules $R\left\{S, S^{-1}\right\} / \mathcal{L}$ for principal left ideals $\mathcal{L}$ other than those generated by a monic linear "polynomial" in $S$. In particular, which such modules appear as dense submodules of $L^{2}(\mathbb{R})$, and what subsequent analytic theories do they yield? One might also consider $Q\left\{S, S^{-1}\right\}$-modules, where $Q$ is the field of fractions of $R$. Alternatively, as in the $p$-adic case, the ring $R$, which 
is a union of polynomial rings, might be replaced by a corresponding union of (formal) power series rings in order to mimic the classical MRA theory wherein the scaling equation may express a scaling function $\phi(x)$ as an infinite series of translates of $\phi(2 x)$ rather than a finite sum, as we have imposed. In the same vein, as noted in Section 3, the ring $R\left\{S, S^{-1}\right\}$ is linked by analogy to the theory of Drinfeld modules and to differential algebra - these connections might be investigated more thoroughly for potential cross-fertilization.

4. Applications. It was not the intent of this paper to provide new applications of multiresolution analyses, but rather to lay some mathematical foundations for possible future uses of WMAs in settings where, for instance, orthogonal forms are not available. It might be desirable, for example, to pursue WMAs over finite fields and their application to codes, monochrome image processing, etc. One measure of the success of this approach will therefore lie in advances that accrue from the algebraic perspective espoused herein.

\section{Appendix: WMAs over $\mathbb{R}$}

We include a proof that every nonzero $\mathbb{R} B_{0}$-module contained in $L^{2}(\mathbb{R})$ is infinite dimensional. Thus the results for infinite-dimensional WMAs in Sections 4 and 5 apply to every WMA in $L^{2}(\mathbb{R})$. Although this fact may be proved by topological/analytic means, it may be nicely deduced from the basic theory of modules over P.I.D.s.

Proposition 7.1. If $V$ is a nonzero subspace of $L^{2}(\mathbb{R})$ such that $f(x-b) \in V$ for every $f \in V$ and $b \in \mathbb{Z}$, then $V$ is infinite dimensional.

Proof. Assume by way of contradiction that $V$ is finite dimensional, so we may assume that $V$ is an irreducible $\mathbb{R} B_{0}$-module. By previous remarks on the structure of $\mathbb{R} B_{0}, V$ is isomorphic to $\mathbb{R}[T] /(p(T))$, for some monic irreducible polynomial $p(T)$. In particular, $V$ is either one or two dimensional.

If $p(T)=T-\alpha$ for some $\alpha \in \mathbb{R}$ and $f$ is a generator for $V$, then $f(x-1)=\alpha f(x)$ for all $x \in \mathbb{R}$. In this case

$$
\int_{0}^{1} f(x-n)^{2} d x=\int_{-n}^{1-n} f(u)^{2} d u=\alpha^{2 n} \int_{0}^{1} f(x)^{2} d x .
$$

Since $f \in L^{2}(\mathbb{R}), \lim _{n \rightarrow \pm \infty} \int_{-n}^{1-n} f(u)^{2} d u=0$. But $\alpha \neq 0$ since $\tau$ is invertible and so $\int_{0}^{1} f(x)^{2} d x=0$. Translating this over all intervals $[k, k+1]$ for $k \in \mathbb{Z}$ shows that $\|f\|_{2}^{2}=0$, a contradiction.

Consider now when $p(T)=(T-\alpha)(T-\bar{\alpha})$ is an irreducible quadratic, for some complex number $\alpha$. Writing $\alpha=r e^{i \theta}$ there is a basis $\left\{f_{1}, f_{2}\right\}$ of $V$ such that with respect to this basis $\tau$ has matrix

$$
[\tau]=r\left(\begin{array}{cc}
\cos \theta & -\sin \theta \\
\sin \theta & \cos \theta
\end{array}\right) .
$$

It follows from De Moivre's formulas that

$$
\begin{aligned}
f_{1}(x-n)^{2}+f_{2}(x-n)^{2} & =\left(\tau^{n} f_{1}(x)\right)^{2}+\left(\tau^{n} f_{2}(x)\right)^{2} \\
& =r^{2 n}\left(\cos ^{2} n \theta+\sin ^{2} n \theta\right) f_{1}(x)^{2}+r^{2 n}\left(\sin ^{2} n \theta+\cos ^{2} n \theta\right) f_{2}(x)^{2} \\
& =r^{2 n}\left(f_{1}(x)^{2}+f_{2}(x)^{2}\right) .
\end{aligned}
$$


As before, integrating over unit intervals and taking the limit as $n \rightarrow \pm \infty$ shows $\left\|f_{1}\right\|_{2}^{2}+\left\|f_{2}\right\|_{2}^{2}=0$, a contradiction. This completes the proof.

\section{ACKNOWLEDGMENT}

The author thanks Gagan Mirchandani and Dan Rockmore for their invaluable assistance with this work. He also thanks the AFOSR for its grant support. Thanks also to the referee for numerous suggestions and references that have significantly improved the paper.

\section{REFERENCES}

[1] J. Benedetto and R. Benedetto, A wavelet theory for local fields and related groups, submitted for publication.

[2] R. Benedetto, Examples of wavelets over local fields, Contemporary Mathematics (2003).

[3] R. Bernanardini and J. Kovačević, Local orthogonal bases I and II, Multidim. Syst. Signal Process. 7 (1996), 331-370 and 371-400.

[4] O. Bratteli and P. Jorgensen, Isometries, Shifts, Cuntz Algebras and Multiresolution Wavelets of Scale N, Integral Equations Operator Th. 28 (1997), 382-443. MR.1465320 (99k:46094b)

[5] G. Chirikjian and A. Kyatkin, Engineering Applications of Noncommutative Harmonic Analysis, CRC Press, 2000. MR 1885369 (2003g:42001)

[6] I. Daubechies, Ten Lectures on Wavelets, CBMS-NSF Regional Conf. Series in Applied Math., SIAM, 1992. MR:1162107 (93e:42045)

[7] D. Dummit and R. Foote, Abstract Algebra, Third Edition, John Wiley \& Sons, Inc, NJ, 2004.

[8] K. Flornes, A. Grossman, M. Holschneider and B. Torrésani, Wavelets on discrete fields, Appl. Comput. Harmon. Anal. 2 (1994), 137-146. MR.1310638 (95h:42031)

[9] R. Foote, G. Mirchandani, D. Rockmore, D. Healy and T. Olson, A wreath product group approach to signal and image processing: part I-multiresolution analysis, IEEE Trans. on Signal Processing 48 (2000), 102-132. MR.1736279 (2001c:94007)

[10] D. Goss, Basic Structures of Function Field Arithmetic, vol. 35, Ergebnisse der Mathematik und ihrer Grenzgebiete, Springer-Verlag, NY, 1996. MR1423131 (97i:11062)

[11] A. Grossmann, Wave functions on subgroups of affine canonical transformations, Resonances —Models and Phenomena, vol. 211, Lecture Notes in Phys., 1984, pp. 128-138. MR0777335 (86f:81042)

[12] A. Grossmann and J. Morlet, Decomposition of Hardy functions into square integrable wavelets of constant shape, SIAM J. Math. Anal. 15 (1984), 723-736. MR0747432 (85i:81146)

[13] A. Grossmann, J. Morlet and T. Paul, Transforms associated to square integrable group representations, I and II, J. Math. Phys., and Ann. Inst. H. Poincaré Phys. Théor., 26, 45 (1985, 1986), 2473-2479 and 293-309 resp. MR0803788(86k:22013), MR0868528(88d:22011)

[14] M. Kirby, Geometric Data Analysis, John Wiley \& Sons, Inc, NJ, 2001. MR.1874451 (2003h:94002)

[15] A. Kyatkin and G. Chirikjian, Algorithms for fast convolutions on motion groups, Appl. Comp. Harm. Anal. 9 (2000), 220-241. MR1777127 (2001g:65177)

[16] S. Mallat, Multiresolution approximations and wavelet orthonormal bases of $l^{2}$, Trans. Amer. Math. Soc. 315 (1989), 69-87. MR1008470 (90e:42046)

[17] D. Maslen, Sampling of functions and sections for compact groups, Modern Signal Processing, D. Rockmore and D. Healy, eds., Cambridge University Press, Cambridge, to appear.

[18] D. Maslen, Efficient computation of Fourier transforms on compact groups, J. Fourier Anal. Appl. 1 (1998), 19-52. MR1650948 (99k:43002)

[19] D. Maslen and D. Rockmore, The Cooley-Tukey FFT and group theory, Notices Amer. Math. Soc. 10 (2001), 1151-1160. MR1861656 (2002k:20022)

[20] D. Maslen and D. Rockmore, Generalized FFTs-a survey of some recent results, Groups and Computation, II (New Brunswick, NJ, 1995), Amer. Math. Soc., Providence, RI, 1997, pp. 183-237. MR.1444138 (98k:20020)

[21] G. Mirchandani, R. Foote, D. Rockmore, D. Healy and T. Olson, A wreath product group approach to signal and image processing: part II-convolution, correlation, and applications, IEEE Trans. on Signal Processing 48 (2000), 749-767. MR1765933 (2001f:94003) 
[22] M. Özaydin and T. Przebinda, Platonic orthonormal wavelets, Appl. Comput. Harmon. Anal. 4 (1997), 351-365. MR 1474094 (99c:42071)

[23] D. Rockmore, Some applications of generalized FFTs, Proceedings of the DIMACS Workshop on Groups and Computation, June 7-10, 1995, L. Finkelstein and W. Kantor, eds., 1997, pp. 329-369. MR.1444144 (98e:65116)

[24] A. Silberger, Introduction to Harmonic Analysis on Reductive p-adic Groups, vol. 23, Mathematical Notes, Princeton University Press, Princeton, NJ, 1979. MR.0544991 (81m:22025)

[25] G. Strang and T. Nguyen, Wavelets and Filter Banks, Addison-Wesley, NY, 1996. MR:1411910 (98b:94003)

[26] B. Yazici, Group invariant methods in signal processing, Proc. Conf. on Information Science and Systems, Johns Hopkins Univ., MD, 1997.

[27] B. Yazici, Stocastic deconvolution over groups, IEEE Trans. in Info. Th., to appear.

Department of Mathematics and Statistics, University of Vermont, Burlington, VerMONT 05405

E-mail address: foote@math.uvm.edu 